\title{
Harmonic and modal frequency discrimination in time domain operational modal analysis
}

\author{
V.H. Vu ${ }^{1}$, M. Thomas ${ }^{1, \text { a }}$ And F. LAfleuR ${ }^{2}$ \\ 1 École de Technologie Supérieure, 1100 Notre Dame West, Montréal, Qc, H3C 1K3, Canada \\ 2 Institut de recherche d'Hydro Québec, 1800 Bl. Lionel-Boulet, Varennes, J3X 1S1, Canada
}

Received 17 June 2013, Accepted 9 December 2013

\begin{abstract}
An algorithm of operational modal analysis in time domain is presented for the discrimination between structural modes, harmonic excitations and spurious modes. A classification factor is developed and based on the construction of the damped signal- to- noise ratio which separates the physical modes from the numerical ones. The harmonic excitations are then recognized at very high power indexes and zero damping. Furthermore, after the modal discrimination, the harmonic spectrum and the estimated frequency response function can be derived. The method can be updated in the time domain either by a short time sliding window or a recursive technique to provide the modal monitoring in both stationary and non-stationary process. An industrial application on a hydro-electrical generator is discussed to identify the machine frequencies under operational working conditions.
\end{abstract}

Key words: Modal identification / mode separation / recursive analysis / short-time modeling / non-stationary vibration / hydro-electric generator

\section{Introduction}

Operational modal analysis has become nowadays an efficient technique for characterization and assessment of machines and structures in vibration. The development of advanced algorithms allows for a robust and accurate analysis in order to insure the productivity and safety. A need appeared for the development of a modal based structural monitoring technique while the machine is always in normal working condition by using an operational modal analysis, especially in rotating machines where the operational vibration can be an important source of failure. As the machine is in working condition, the discrimination of the structural modes from the harmonic excitations and noises and the monitoring of each mode with respect to time are not a trivial task, especially for unstationary systems. In the frequency domain, Brinker et al. [1] presented the frequency domain decomposition method with an indicator based on the observation of the probability density functions at each frequency. However, application of the frequency domain to the operational time domain is seen limited. Jacobsen presented some techniques for separating structural modes

a Corresponding author: marc.thomas@etsmtl.ca and harmonics [2]. Those techniques are qualitative and require all a visual judgment on the observation of such spectrum, mode shape plot and/or Modal Assurance Criterion (MAC). In the time domain, the Least Squares Complex Exponential (LSCE) [3] and the Ibrahim Time Domain (ITD) method [4] have been developed for the modal identification. ITD applied the random-decrement technique coupled with a time-domain parameter identification method to process ambient vibration response that can be approximated as a sum of exponential functions. However the selection of specific instants into the signal has been found critical when applying the ITD method. Mohanty et al. [5] have tried to modify those methods to adapt the operational modal analysis in the case where a harmonic component is close to a natural frequency. Actually they have improved the technique to enhance the presence of those modes on the frequency stabilization diagram. However the harmonics frequencies are assumed to be known a priori [5].

In this paper, we present a method to classify the identified modes in order to discriminate the physical modes from the spurious modes and then distinguish the harmonic excitations from the natural frequencies. The method is based on the construction of a factor called 
Damped Modal Signal to Noise ratio (DMSN), obtained from the modal decomposition of the signal into deterministic and stochastic parts.

\section{Modeling by vector autoregressive model (VAR)}

In operational modal analysis, we assume that the excitation is unknown and can be modeled by a Gaussian white noise. As modal analysis is conducted by using several $d$ channels of measurements, synchronized for data acquisition at a sampling period $T_{\mathrm{s}}$, the vector time series such as the Vector AutoRegressive (VAR) or Vector Autoregressive Moving Average (VARMA) could be used for modeling the data. Since the modal parameters are therefore extracted from the AutoRregressive (AR) part of those models and since the excitation is Gaussian, the least squares estimation is non-biased and a vector autoregressive (VAR) model of $p^{\text {th }}$ order and of dimension $d$ has been found sufficient to fit the measured data $[6,7]$ :

$$
\mathbf{y}(t)=\mathbf{\Lambda} \mathbf{z}(t)+\mathbf{e}(t)
$$

where:

$$
\begin{aligned}
& \boldsymbol{\Lambda}=\left[-\mathbf{A}_{1}-\mathbf{A}_{2} \ldots-\mathbf{A}_{p}\right] \text { size } d \times d p \text { is the parameter } \\
& \text { matrix; }
\end{aligned}
$$

$\mathbf{A}_{i}($ size $d \times d)$ is the matrix of parameters relating the output $\mathbf{y}(t-i)$ to $\mathbf{y}(t)$; $\mathbf{z}(t)=\left[\mathbf{y}(t-1)^{\mathrm{T}} \mathbf{y}(t-2)^{\mathrm{T}} \ldots \mathbf{y}(t-p)^{\mathrm{T}}\right]^{\mathrm{T}}($ size $d p \times$ $1)$ is the regressor for the output vector $\mathbf{y}(t)$;

$\mathbf{y}(t-i)($ size $d \times 1 ; i=1: p)$ is the output vector with delay time $i \times T_{\mathrm{s}}$;

$\mathbf{e}(t)$ (size $d \times 1$ ) is the residual vector of all output channels considered as the error of model.

If the data are assumed to be measured in a white noise environment, the least squares estimation may be applied for estimating the model parameters. Taking into account $N$ successive available output vectors of the responses from $\mathbf{y}(k)$ to $\mathbf{y}(k+N-1)(k>p, N>d p+d)$, the model parameters matrix $\boldsymbol{\Lambda}$ and the estimated covariance matrices of the deterministic part $\hat{\mathbf{D}}$ and of the error part $\hat{\mathbf{E}}$ (both of size $d \times d$ ) can be estimated via the computation of the QR factorization as follows $[6,8]$ :

$$
\begin{aligned}
& \boldsymbol{\Lambda}=\left(\mathbf{R}_{12}^{\mathrm{T}} \mathbf{R}_{11}\right) \cdot\left(\mathbf{R}_{11}^{\mathrm{T}} \mathbf{R}_{11}\right)^{-1}=\left(\mathbf{R}_{11}^{-1} \mathbf{R}_{12}\right)^{\mathrm{T}} \\
& \hat{\mathbf{D}}=\frac{1}{N} \mathbf{R}_{12}^{\mathrm{T}} \mathbf{R}_{12} \\
& \hat{\mathbf{E}}=\frac{1}{N} \mathbf{R}_{22}^{\mathrm{T}} \mathbf{R}_{22}
\end{aligned}
$$

In these formulas, $\mathbf{R}_{11}($ size $d p \times d p), \mathbf{R}_{12}($ size $d p \times d)$ and $\mathbf{R}_{22}$ (size $d \times d$ ) are sub-matrices of the upper triangular factor $\mathbf{R}$ (size $N \times d p+d$ ) derived from the $\mathrm{QR}$ factorization of the data matrix as follows:

$$
\mathbf{K}=\mathbf{Q} \times \mathbf{R}
$$

where $\mathbf{Q}$ (size $N \times N)$ is an orthogonal matrix (that is $\left.\mathbf{Q} \times \mathbf{Q}^{\mathrm{T}}=\mathbf{I}\right), \mathbf{R}$ has the form:

$$
\mathbf{R}=\left[\begin{array}{cc}
\mathbf{R}_{11} & \mathbf{R}_{12} \\
\mathbf{0} & \mathbf{R}_{22} \\
\mathbf{0} & \mathbf{0}
\end{array}\right]
$$

and data matrix $\mathbf{K}$ of size $N \times d p+d$ is constructed from $N$ successive samples:

$$
\mathbf{K}=\left[\begin{array}{cc}
\mathbf{z}(t)^{\mathrm{T}} & \mathbf{y}(t)^{\mathrm{T}} \\
\mathbf{z}(t+1)^{\mathrm{T}} & \mathbf{y}(t+1)^{\mathrm{T}} \\
\cdots & \cdots \\
\mathbf{z}(t+N-1)^{\mathrm{T}} & \mathbf{y}(t+N-1)^{\mathrm{T}}
\end{array}\right]
$$

Once the model's parameters matrix has been estimated, the modal parameters can directly be identified from the eigen-decomposition of the state matrix $\boldsymbol{\Pi}$ (size $d p \times$ $d p)$ [8]:

$$
\boldsymbol{\Pi}=\left[\begin{array}{cccccc}
-\mathbf{A}_{1} & & -\mathbf{A}_{2} & \ldots & -\mathbf{A}_{p-1} & -\mathbf{A}_{p} \\
\mathbf{I} & \mathbf{0} & \ldots & \mathbf{0} & \mathbf{0} \\
\mathbf{0} & \mathbf{I} & \ldots & \mathbf{0} & \mathbf{0} \\
\ldots & \ldots & \ldots & \ldots & \ldots & \\
\mathbf{0} & \mathbf{0} & \ldots & \mathbf{I} & \mathbf{0} &
\end{array}\right]
$$

$$
\boldsymbol{\Pi}=\mathbf{L}\left[\begin{array}{cccc}
\lambda_{1} & 0 & 0 & 0 \\
0 & \lambda_{2} & 0 & 0 \\
0 & 0 & \ddots & \vdots \\
0 & 0 & \ldots & \lambda_{d p}
\end{array}\right] \mathbf{L}^{-1}
$$

where $\lambda_{i}$ are discrete eigenvalues and $\mathbf{L}($ size $d p \times d p)$ is the eigenvectors matrix, whose forms can be rewritten as follows for further usage:

$$
\begin{aligned}
& \mathbf{L}=\left[\begin{array}{rrrrr}
\lambda_{1}^{p-1} \mathbf{l}_{1} & \lambda_{2}^{p-1} \mathbf{l}_{2} & \ldots & \lambda_{d p}^{p-1} \mathbf{l}_{d p} \\
\vdots & \vdots & \vdots & \vdots \\
\lambda_{1} \mathbf{l}_{1} & \lambda_{2} \mathbf{l}_{2} & \ldots & \lambda_{d p} \mathbf{l}_{d p} \\
\mathbf{l}_{1} & \mathbf{l}_{2} & \ldots & \mathbf{l}_{d p}
\end{array}\right] \\
& \mathbf{S}=\mathbf{L}^{-1}=\left[\begin{array}{cccc}
\mathbf{S}_{11} & \mathbf{S}_{12} & \ldots & \mathbf{S}_{1 p} \\
\mathbf{S}_{21} & \mathbf{S}_{22} & \ldots & \mathbf{S}_{2 p} \\
\vdots & \vdots & \ddots & \vdots \\
\mathbf{S}_{d p 1} & \mathbf{S}_{d p 2} & \ldots & \mathbf{S}_{d p p}
\end{array}\right]
\end{aligned}
$$

The continuous eigenvalues, natural frequencies, damping ratios and complex modes are derived as follows:

$$
\begin{aligned}
& \text { Eigenvalues: } u_{i}=\frac{\ln \left(\lambda_{i}\right)}{T_{\mathrm{s}}} \\
& \text { Frequencies: } f_{i}=\frac{\sqrt{\boldsymbol{R e}^{2}\left(u_{i}\right)+\boldsymbol{I m}^{2}\left(u_{i}\right)}}{2 \pi} \\
& \text { Damping ratios: } \xi_{i}=-\frac{\boldsymbol{R} \boldsymbol{e}\left(u_{i}\right)}{2 \pi f_{i}} \\
& \text { Complex modes: } \boldsymbol{\Psi}=\left[\begin{array}{llll}
\boldsymbol{\Psi}_{1} & \boldsymbol{\Psi}_{2} & \ldots & \Psi_{d p}
\end{array}\right]=\left[\begin{array}{llll}
\mathbf{I} & \mathbf{0} & \ldots & \mathbf{0}
\end{array}\right] \mathbf{L}
\end{aligned}
$$

\section{Construction of the damped modal signal to noise ratio}

With any model based modal analysis, the signal can be decomposed into a deterministic part and a stochastic 

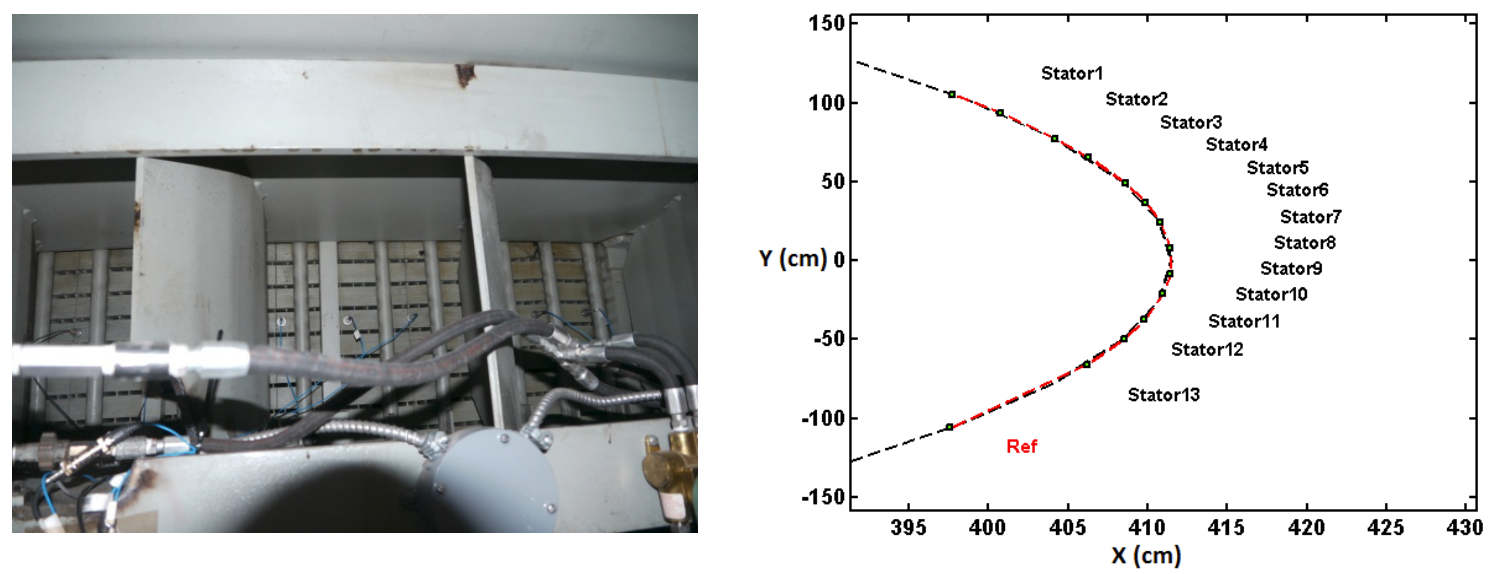

Fig. 1. Sensors configuration on 2D Cartesian coordinates $(\mathrm{cm})$.

part from the eigen-decomposition as follows [8]:

$$
\mathbf{y}(t)=\sum_{i=1}^{d p}\left[\mathbf{l}_{i} \mathbf{s}_{i} \lambda_{i}^{t}+\sum_{j=0}^{t-p} \mathbf{l}_{i} \mathbf{S}_{i 1} \mathbf{e}(t-j) \lambda_{i}^{j+p-1}\right]
$$

where:

$\mathbf{y}(t)$ is the measured sample data at time $t$, of dimension $d \times 1$

$d$ is the number of sensors, or vector dimension

$p$ is the model order in the modeling

$\mathbf{l}_{i}$ are taken from eigenvector matrix $\mathbf{L}$

$\mathbf{s}_{i}$ is the scale factor which can be computed from the initial data as:

$$
\begin{aligned}
\mathbf{s} & =\mathbf{L}^{-1} \mathbf{z}(p+1) \\
\mathbf{z}(p+1)^{\mathrm{T}} & =\left[\mathbf{y}(p)^{\mathrm{T}} \mathbf{y}(p-1)^{\mathrm{T}} \ldots \mathbf{y}(1)^{\mathrm{T}}\right]
\end{aligned}
$$

$\lambda_{i}$ are discrete eigenvalues,

$\mathbf{S}_{i 1}$ are extracted from the inverse eigenvector matrix, $\mathbf{e}(t)$ is the model error vector sampled at time $t$.

It is seen that the first term of equation (12) is the deterministic part and the second one if the stochastic part of the sampled signal. Those parts are the sum from the contribution of all eigenvalues and eigenmodes.

If we consider the contribution of each mode to the deterministic part, we can take the modal power of the discrete signal over the $N$ available sampled data:

$\mathrm{MP}_{i}=\sum_{t=k}^{k+N-1} \mathbf{l}_{i}^{\mathrm{H}} \mathbf{l}_{i}\left|s_{i}\right|^{2}\left|\lambda_{i}\right|^{2(t-k)}=\frac{\mathbf{l}_{i}{ }^{\mathrm{H}} \mathbf{l}_{i}\left|s_{i}\right|^{2}\left(1-\left|\lambda_{i}\right|^{2 N}\right)}{1-\left|\lambda_{i}\right|^{2}}$

Similarly, the contribution of each mode to the stochastic part can be computed in term of the modal variance [8]:

$$
\mathrm{MV}_{i}=\sum_{t=k}^{k+N-1} \mathrm{MV}_{i}^{t}=\frac{{ }_{i}{ }^{\mathrm{H}} \mathbf{l}_{i} \mathbf{L}^{i 1} \hat{\mathbf{E}} \mathbf{S}_{i 1}^{\mathrm{H}}\left[N-\frac{\left|\lambda_{i}\right|^{2}\left(1-\left|\lambda_{i}\right|^{2 N}\right)}{1-\left|\lambda_{i}\right|^{2}}\right]}{1-\left|\lambda_{i}\right|^{2}}
$$

where $\hat{\mathbf{E}}$ is the estimated error matrix of the underlying parametrical model.
Since the frequency and damping of each mode are identified, the factor called Damped Modal Signal to Noise (DMSN) ratio is proposed as follows:

$$
\operatorname{DMSN}_{i}=\frac{\mathrm{MP}_{i}}{\zeta_{i} \mathrm{MV}_{i}}
$$

It appears that the DMSN index is an effective criterion since it includes the stochastic participation in the denominator, and hence, the higher the DMSN, the more evident it characterizes the contribution to the deterministic part. The damping rate on the denominator penalizes the very high damped modes which usually belong to computational modes. The damping rate $\zeta_{i}$ is more usually found from $0.5 \%$ to $7 \%$ in industrial applications when no external damping is added.

The modes are all sorted from the first highest DMSN index, with reasonable damping ratios. Consequently, the harmonic frequencies, if present, are first revealed, and can then be distinguished from the natural frequencies by their close-to-zero damping ratio, and hence a very high DMSN index. The available natural frequencies then follow and the computational frequencies end up with a very low DMSN.

\section{Application to machines in operational conditions}

The method was applied to a real industrial application on a hydro-electrical generator. The test is a part of the project at Hydro Quebec research institute on increasing the power of existing generators [9-11].

It is seen that the hydro electrical generators are of huge dimensions structures which are working under severe electromagnetic excitations. Therefore operational modal analysis are conducted in order to estimate the real structural responses and to identify the excitations [9-12].

Because of the generator huge dimension and sensor limitation, various configurations were designed to measure up to one quarter of the stator by using of a common reference sensor. Figure 1 shows a configuration with a 


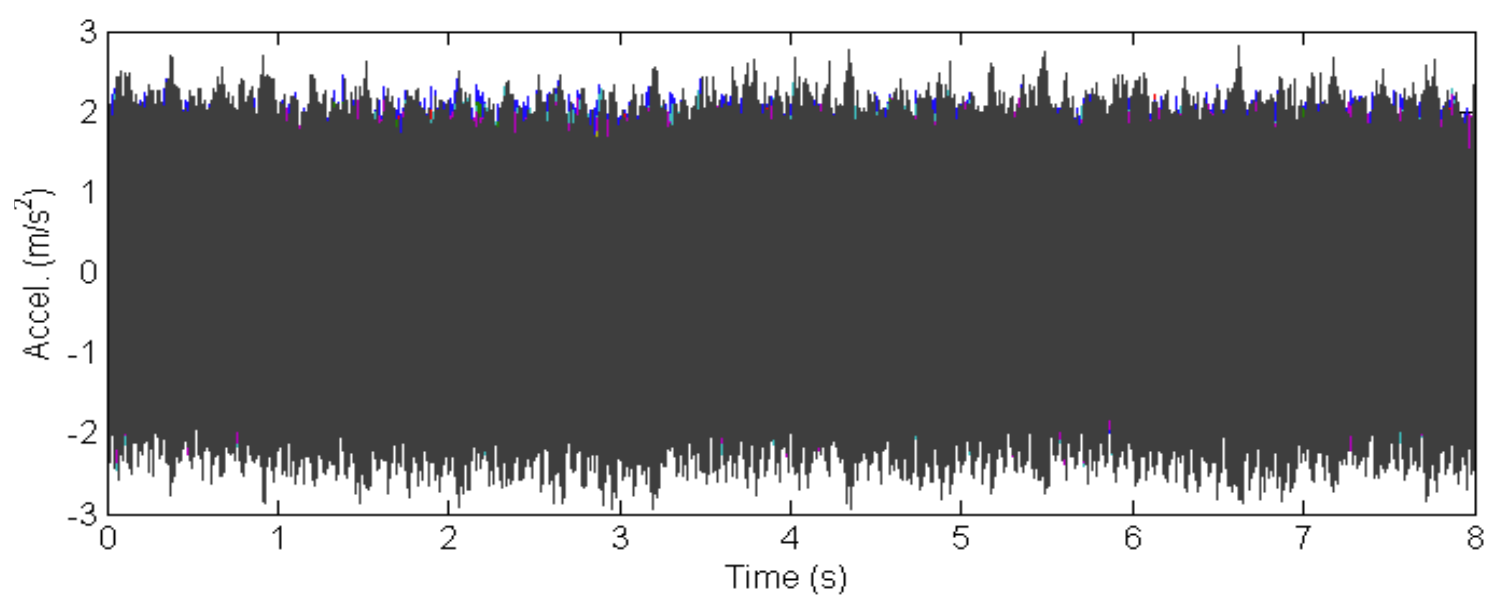

Fig. 2. Stationary signal.

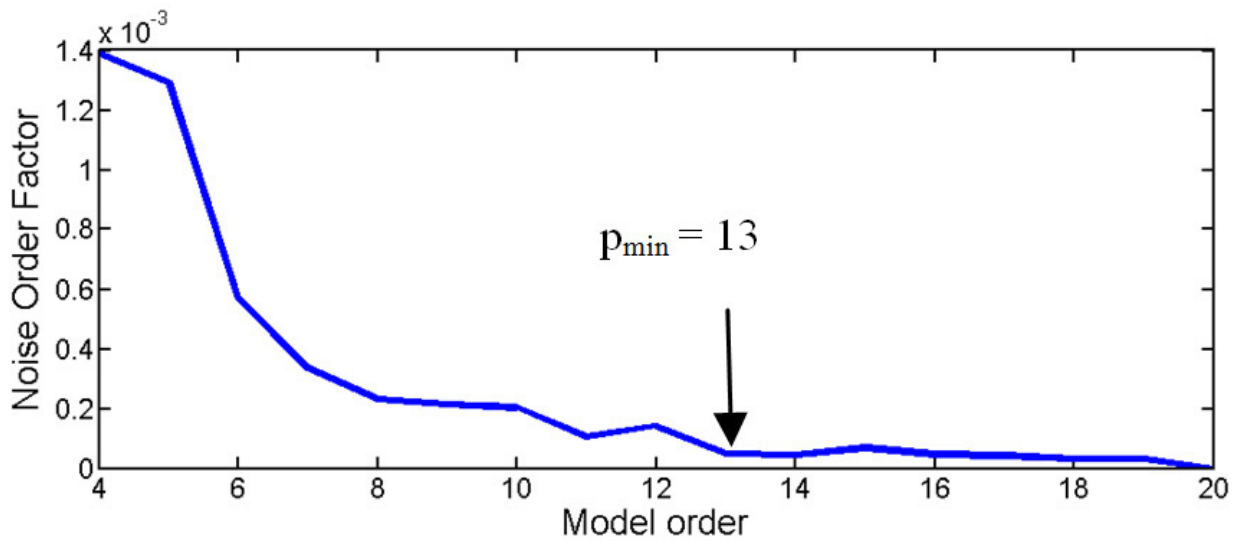

Fig. 3. NOF.

typical 14 accelerometers mount on the perimeter of the stator. Vibration data are acquired at $4096 \mathrm{~Hz}$ rate.

\subsection{Stationary vibration}

The first test is done when the machine was working in stationary condition at a power rated 23 MW. Figure 2 presents the time response of all the sensors.

A Noise rate Order Factor (NOF) [6] had been previously developed to identify the minimum necessary order when decreasing as shown in Figure 3. The order is selected when NOF decreases drastically. It was found that the NOF factor is very robust regardless the data length when the later is long enough and that the model VAR can fit with a lower model order when higher a number " $d$ " of channels are used. However, it is an approximate criterion that indicates in this study that an efficient order between 13 and 20 can be used for the identification. From the damping criteria, the first 20 frequencies have been selected as real frequencies and the DMSN indicates that the 5 first one comes from harmonic components.
Table 1 shows the identification of those 20 modes with frequencies, damping ratios and corresponding acceleration amplitudes (m.s $\left.{ }^{-2}\right)$.

From Table 1 , it is found that there are 5 dominant electromagnetic harmonics in the vibration that correspond to the highest DMSN and amplitudes. These frequencies can be distinguished at the significant change of the DMSN slope. It is also found that except the electromagnetic harmonics of $60 \mathrm{~Hz}$ (especially, the harmonics of the even multiple of the electric fundamental frequency $(120 \mathrm{~Hz}, 240 \mathrm{~Hz} .$. ) $)$ with zero closed damping rates, there are 4 frequencies that are not harmonics and are the natural frequencies: 728.3, 740.3, 1480.6 and $1599.4 \mathrm{~Hz}$. Figure 5 shows the Operating Deflection Shape (ODS) of the four most excited harmonics and two mode shapes. From the number of waveforms identified from the measured arc, it is convenient to generate the total number of waveforms on the whole perimeter for each frequency. It is also seen that the closeness to a natural frequency explains why the two first harmonics at $720 \mathrm{~Hz}$ and $1560 \mathrm{~Hz}$ the most excited. The ODS of the $720 \mathrm{~Hz}$ is found similar to the mode shape of $728 \mathrm{~Hz}$ (presented out of phase). 


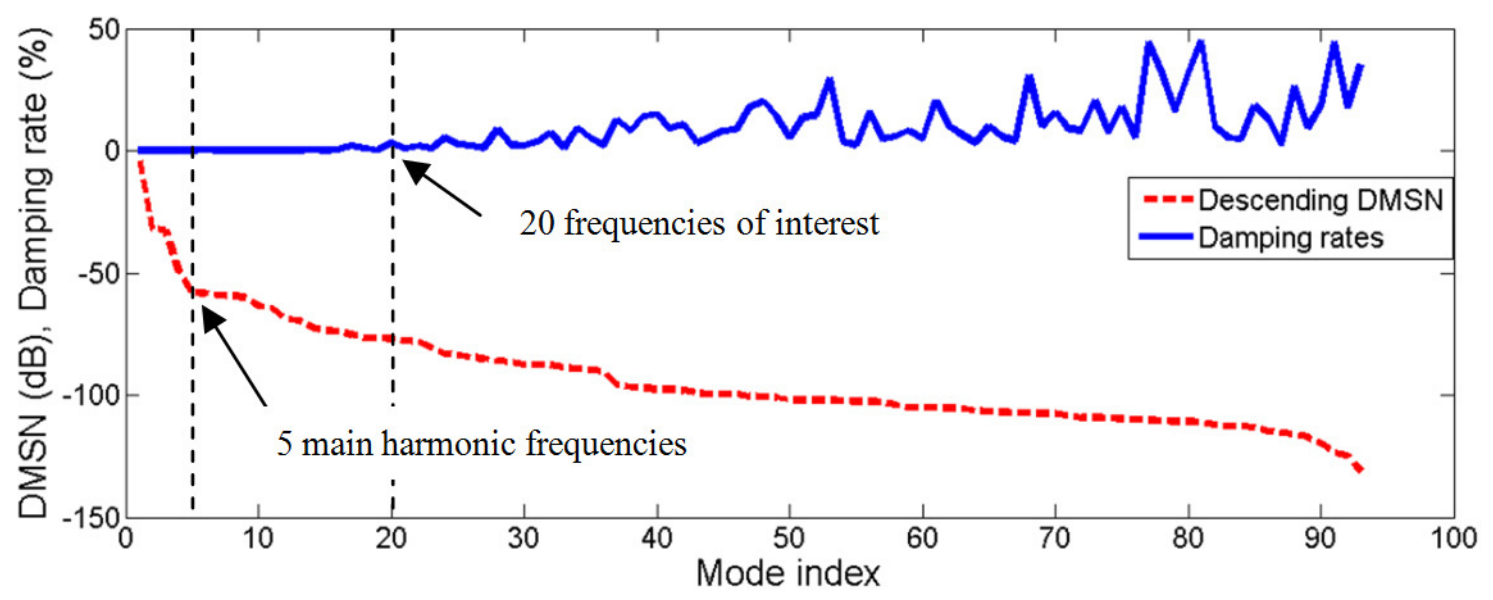

Fig. 4. DMSN.

Table 1. Modal parameters identification.

\begin{tabular}{cccccccc}
\hline Mode & $\begin{array}{c}\text { Frequency } \\
(\mathrm{Hz})\end{array}$ & $\begin{array}{c}\text { Damping rate } \\
(\%)\end{array}$ & $\begin{array}{c}\text { Amplitude } \\
\left(\mathrm{m} . \mathrm{s}^{-2}\right)\end{array}$ & $\begin{array}{c}\text { Mode } \\
\text { Frequency } \\
(\mathrm{Hz})\end{array}$ & $\begin{array}{c}\text { Damping rate } \\
(\%)\end{array}$ & $\begin{array}{c}\text { Amplitude } \\
\left(\mathrm{m}_{\mathrm{s}} \mathrm{s}^{-2}\right)\end{array}$ \\
\hline 1 & 719.9 & 0.0 & 0.33 & 11 & 120.0 & 0.1 & 0.02 \\
2 & 1559.8 & 0.0 & 0.03 & 12 & 839.7 & 0.0 & 0.00 \\
3 & 1439.8 & 0.0 & 0.03 & 13 & 360.1 & 0.1 & 0.01 \\
4 & 1199.9 & 0.0 & 0.01 & 14 & 1599.4 & 0.5 & 0.00 \\
5 & 599.9 & 0.1 & 0.02 & 15 & 1679.7 & 0.1 & 0.00 \\
6 & 728.3 & 0.4 & 0.01 & 16 & 1799.7 & 0.3 & 0.00 \\
7 & 1080.0 & 0.1 & 0.00 & 17 & 740.3 & 1.9 & 0.00 \\
8 & 960.0 & 0.1 & 0.00 & 18 & 1480.6 & 1.0 & 0.00 \\
9 & 1319.9 & 0.0 & 0.00 & 19 & 1919.5 & 0.1 & 0.00 \\
10 & 479.8 & 0.1 & 0.01 & 20 & 778.7 & 3.1 & 0.00 \\
\hline
\end{tabular}

\subsection{Non-stationary vibration}

The machine was also monitored during a start-up regime to examine the non stationary vibration. Figure 6 presents the signal data of all 14 sensors from the beginning to gain the operational power at $23 \mathrm{MW}$.

Using the sliding window technique, a short time AR (STAR) method was applied onto the signal with window length 2000 samples $(0.5 \mathrm{~s})$ without overlapping [12,13]. Figure 7 shows the monitoring of the AR model order, frequencies and damping rates computed at an optimal order with respect to time.

It is seen that this optimal AR model order is found to 8 or 9 for the modal identification. The frequencies of all harmonics and structural modes are accurately identified and monitored. A slight increasing in frequencies, mostly at high frequencies can be detected at the beginning of the start up before stabilized to a stationary regime. The damping ratios can also be tracked for each mode but with a greater uncertainty [14]. The plot of all modes together shows that the damping rates are found less than $5 \%$. If one looks close up to the two structural modes, it is seen that those modes can also be monitored and distinctly discriminated from the closer harmonic, as seen in Figures 8 and 9.

Figures $8 \mathrm{a}$ and $9 \mathrm{a}$ show very clearly the changes in frequencies during the non-stationary vibration. However, the uncertainty on damping identification is greater [14]. Figure $8 \mathrm{~b}$ shows that the damping rate of the structural mode at $728 \mathrm{~Hz}$ is about $0.5 \%$ while Figure $9 \mathrm{~b}$ reveals that the damping rate is close to $1.2 \%$ at $1599 \mathrm{~Hz}$.

\section{Conclusion}

A technique for modal identification and discrimination is presented for operational modal analysis and monitoring purpose. A Damped Modal Signal to Noise ratio (DMSN) is found a reliable index for classification of frequencies and modal parameters and hence for the distinction of structural modes and harmonics excitations from spurious frequencies. It is found from a real industrial application that this technique can be used in offline analysis or online processing with both stationary and non stationary vibrations. The modal parameters of each mode can be tracked and monitored with a sufficient precision. 


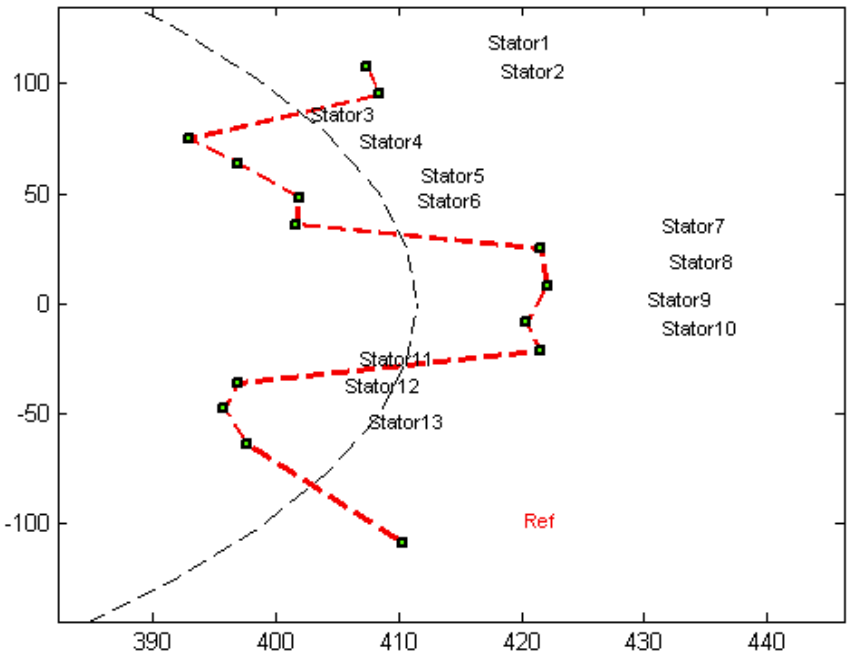

a) ODS $720 \mathrm{~Hz}$
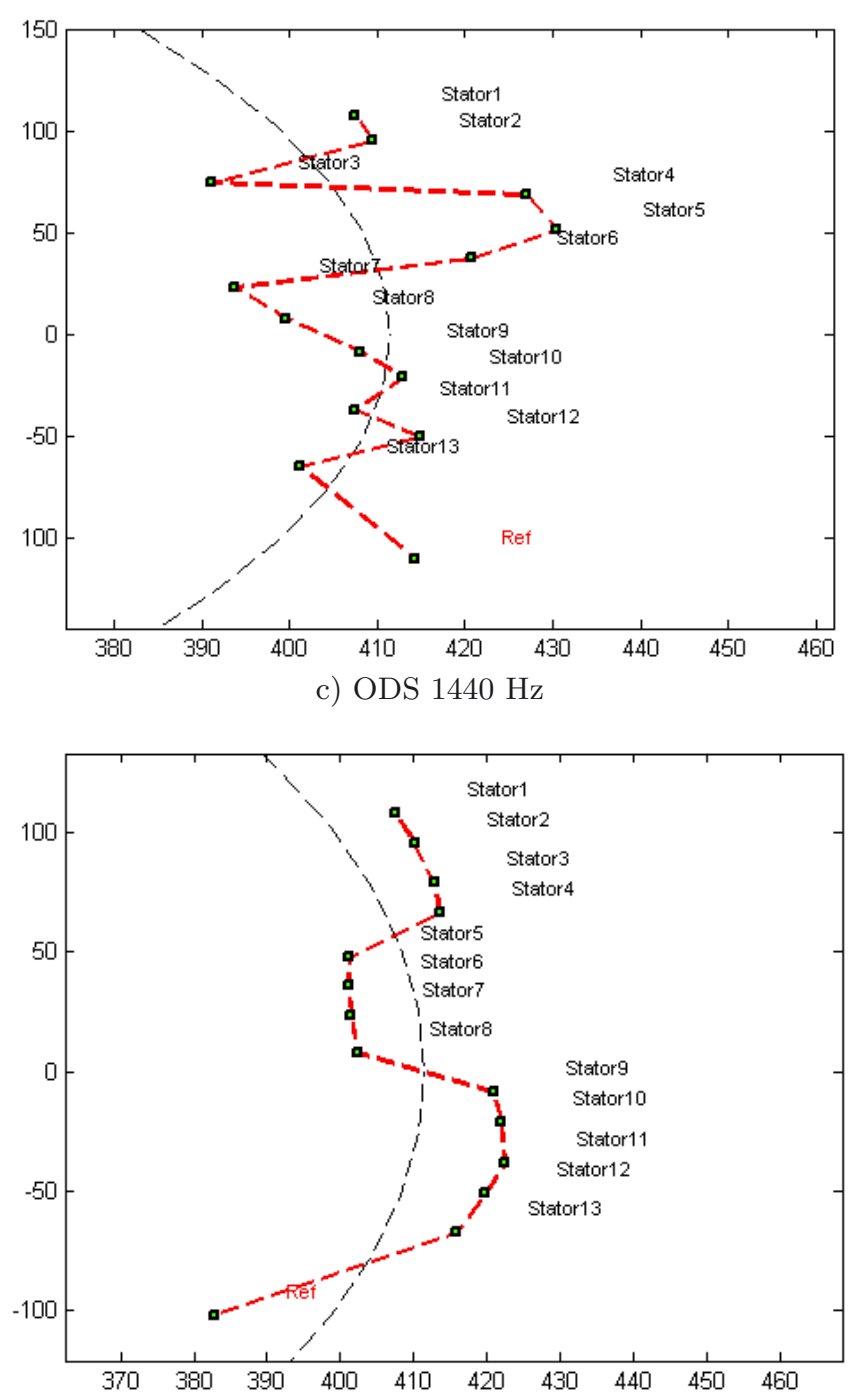

e) Mode shape $728 \mathrm{~Hz}$

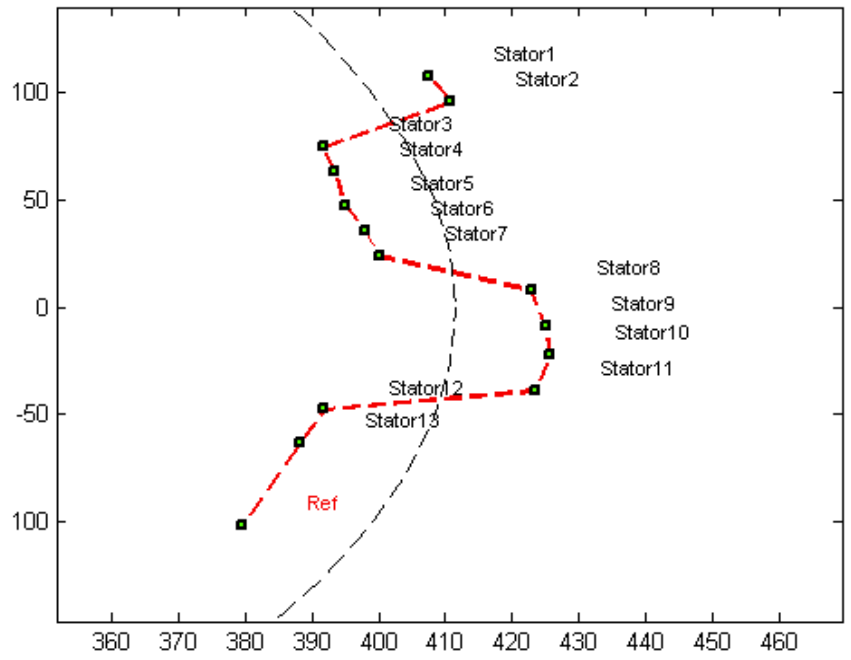

b) ODS $1560 \mathrm{~Hz}$
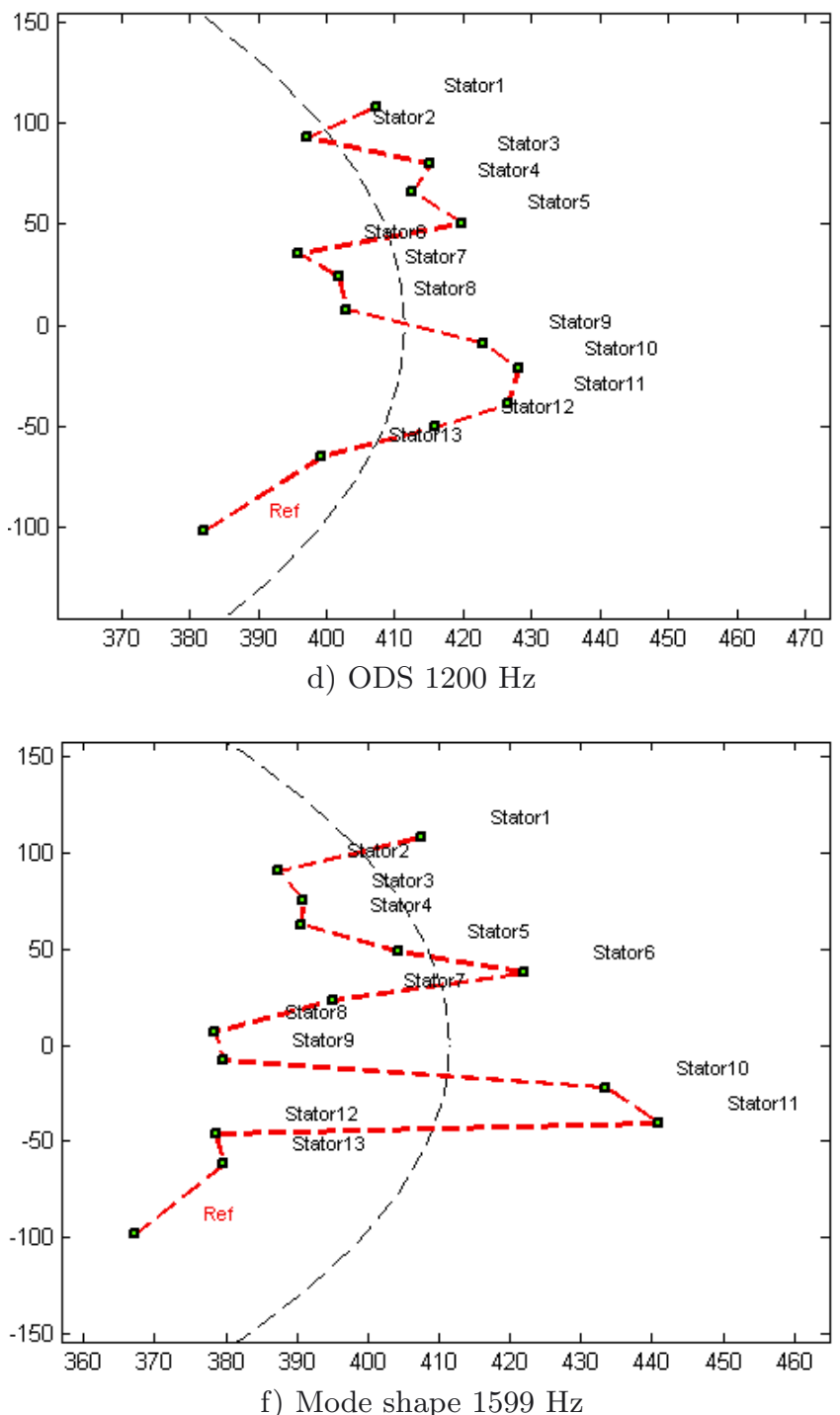

f) Mode shape $1599 \mathrm{~Hz}$

Fig. 5. ODS and mode shapes. 


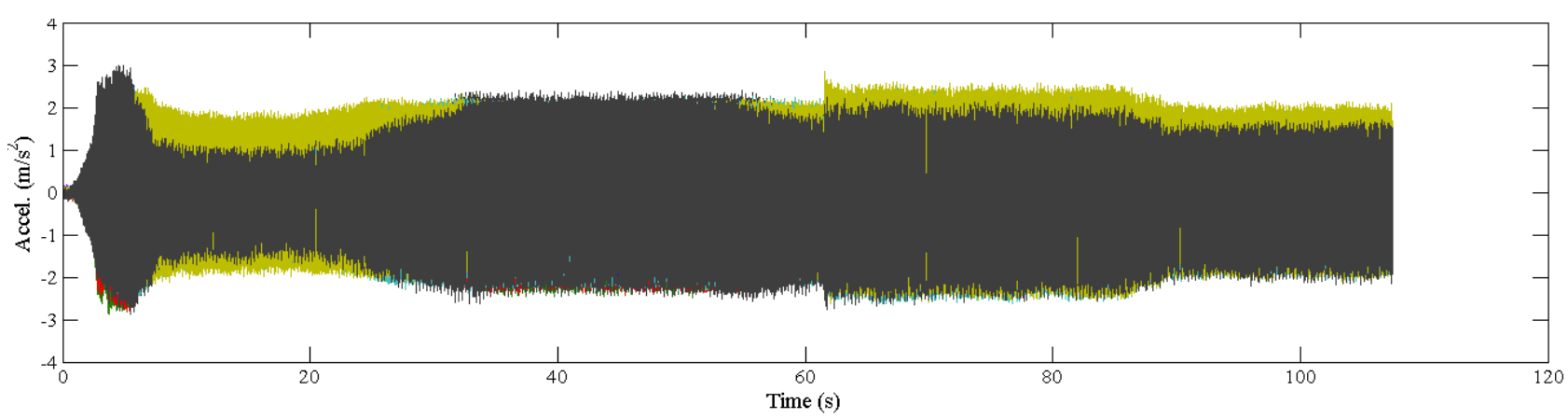

Fig. 6. Start-up non stationary signal.

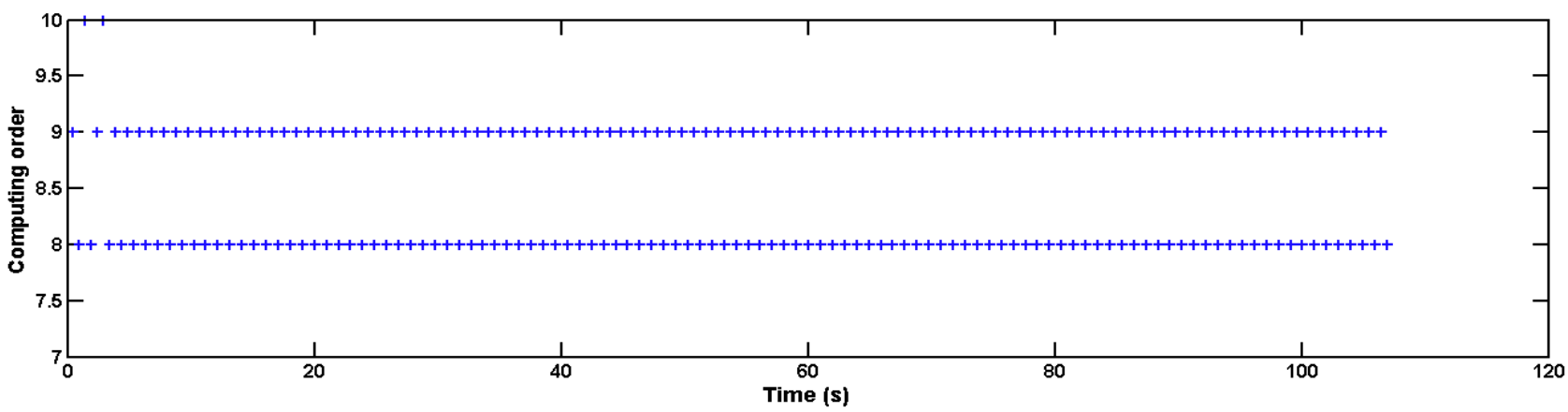

a) Autoregressive optimal model order

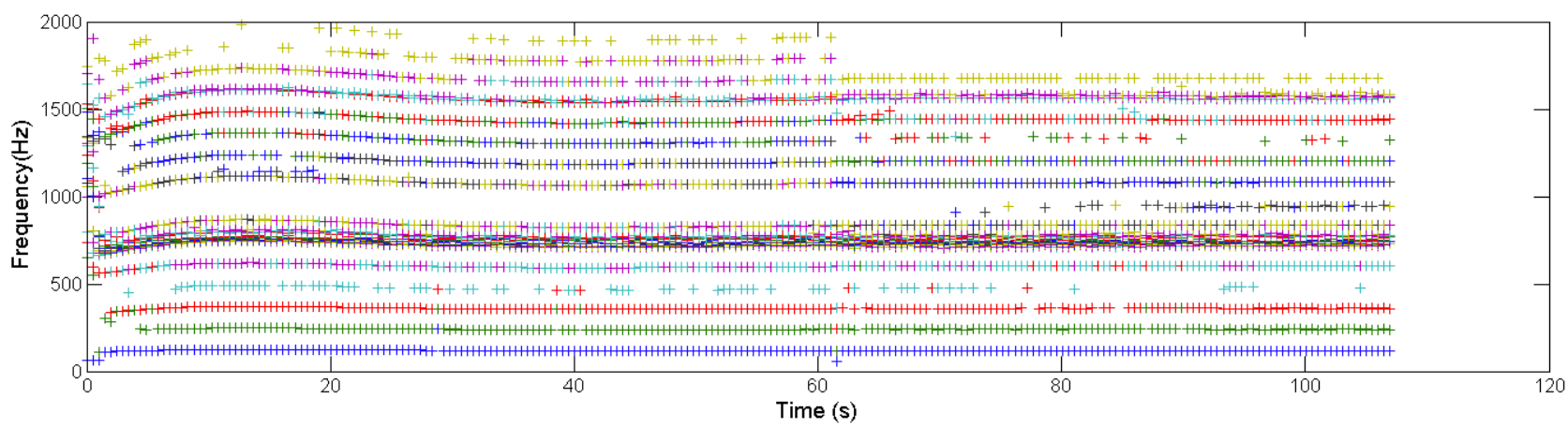

b) Frequencies

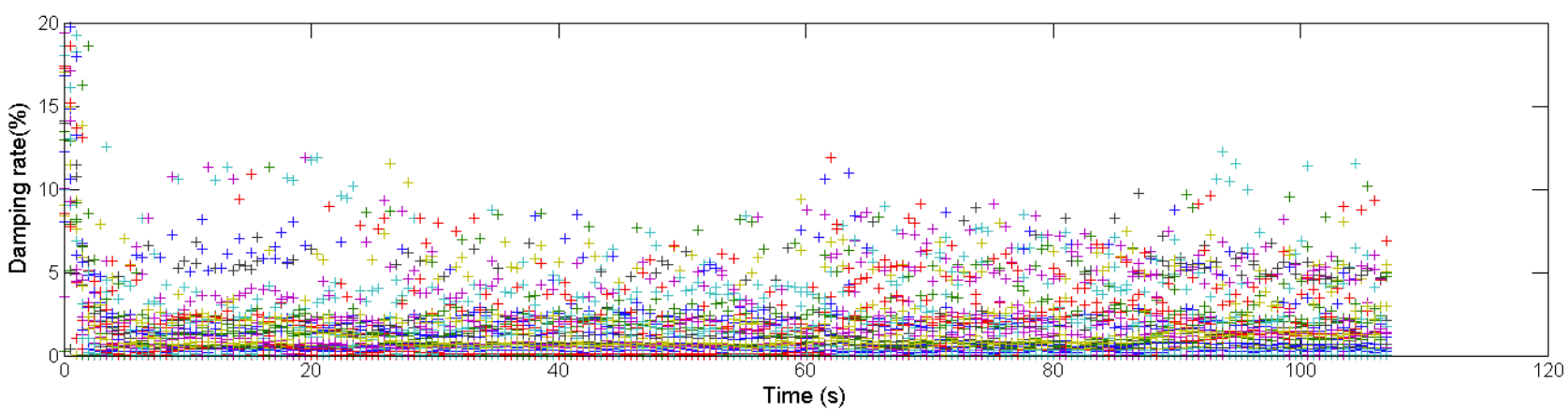

c) Damping ratios

Fig. 7. Online modal monitoring. 


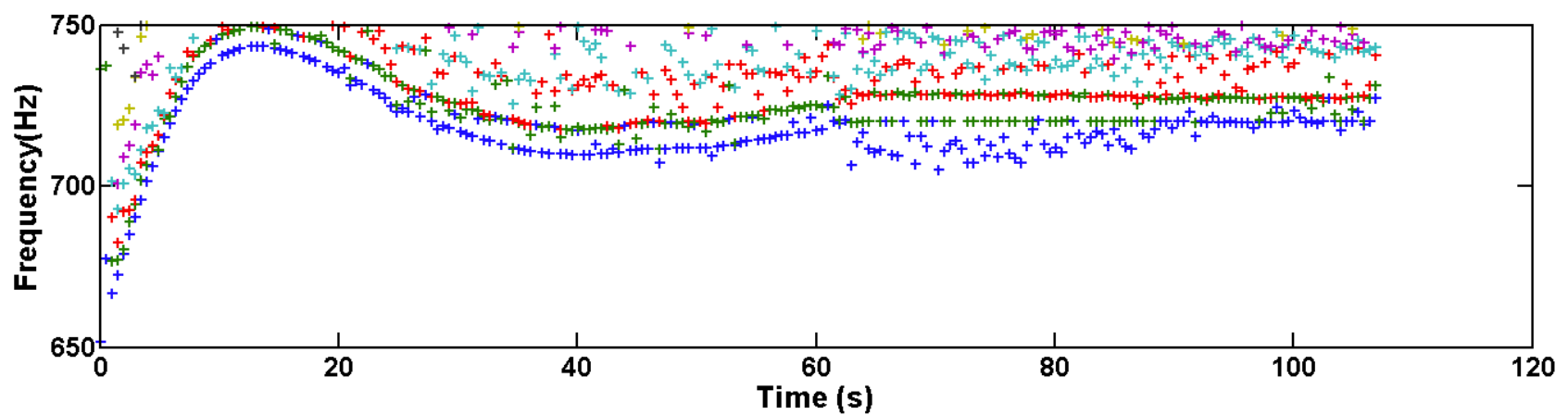

a) Frequency

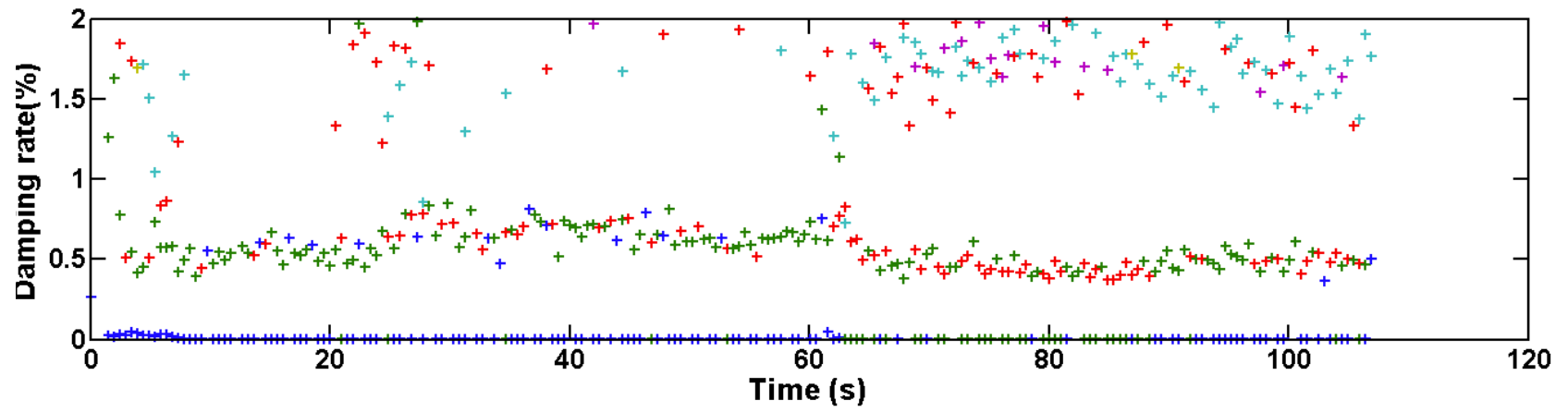

b) Damping rate

Fig. 8. Modal discrimination at $720 \mathrm{~Hz}$.

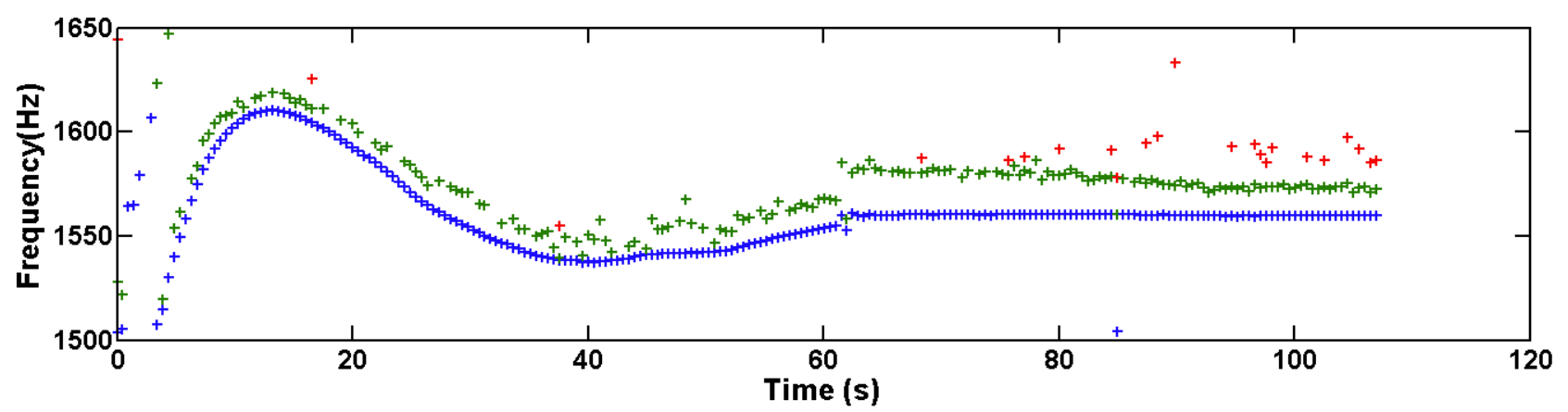

a) Frequency

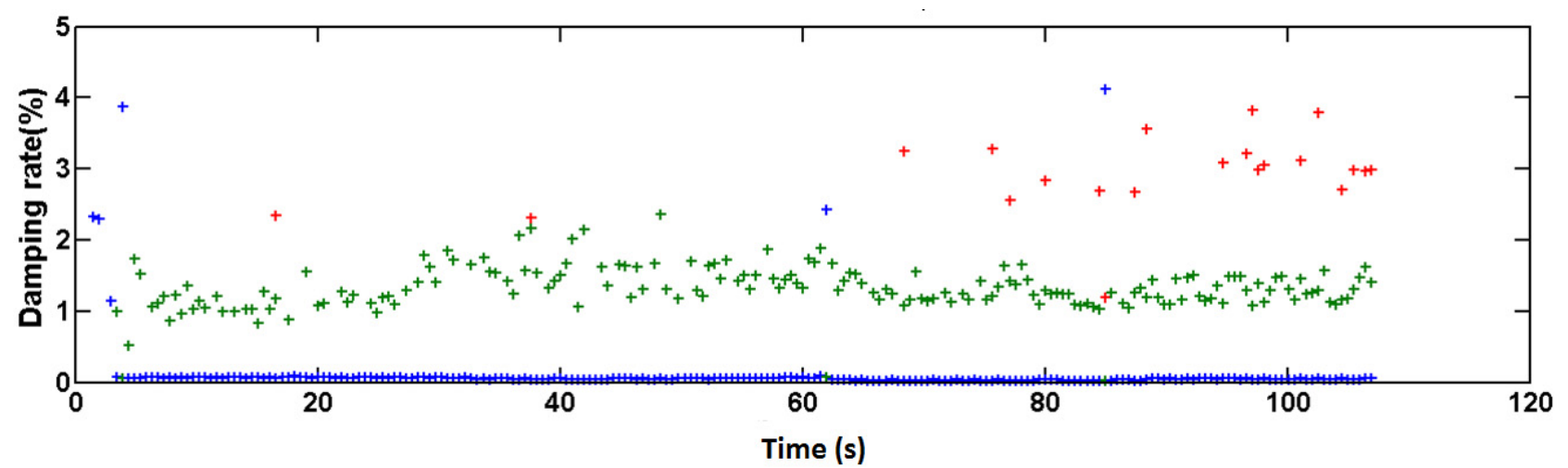

b) Damping rate

Fig. 9. Modal discrimination at $1560 \mathrm{~Hz}$. 
Acknowledgements. The authors would like to thank researchers and technicians at IREQ (Institut de recherche d'Hydro Québec) for their collaboration and support on this research project. The authors would also like to thank the programs MITACS Canada and FQRNT for their financial supports in this study.

\section{References}

[1] R. Brincker, P. Andersen, N. Møller, An Indicator for Separation of Structural and Harmonic Modes in OutputOnly modal Testing, IMAC XVIII, 2000, 8p

[2] N.J. Jacobsen, Eliminating the Influence of Harmonic Components in Operational Modal Analysis, IMAC XXV, 2007, $11 \mathrm{p}$

[3] D.L. Brown, R.J. Allemang, R. Zimmerman, M. Mergeay, Parameter Estimation Techniques for Modal Analysis, 7th International Seminar on Modal Analysis, Katholieke Universiteit Leuven, Belgium, 1985

[4] S.R. Ibrahim, E.C. Mikulcik, A method for Direct Identification of Vibration Parameters from the Free Response, The Shock and Vibration Bulletin 47 (1977) $183-198$

[5] P. Mohanty, D.J. Rixen, Operational modal analysis in the presence of harmonic excitation, J. Sound Vib. 270 (2004) 93-109

[6] V.H. Vu, M. Thomas, A.A. Lakis, L. Marcouiller, Operational modal analysis by updating autoregressive model, Mechanical systems and signal processing (MSSP), Elsevier, 25 (2011) 1028-1044
[7] V.H. Vu, M. Thomas, F. Lafleur, L. Marcouiller, Towards an automatic spectral and modal identification from operational modal analysis. J. Sound Vib. Elsevier. 332 (2013) 213-227

[8] S.M. Pandit, 1991, Modal and spectrum analysis: data dependent systems in state space. New York, J. Wiley and Sons, 415 p.

[9] F. Lafleur, V.H. Vu, M. Thomas, Oct 2011, Case study of operational modal analysis of a large hydroelectric generator, Proceedings of the 29th Seminar on machinery vibration, Canadian Machinery Vibration Association, St John (NB, Canada), 11 pages

[10] V.H. Vu, M. Thomas, F. Lafleur, Oct 2011, Operational Modal Monitoring of High Power Hydro-electrical Generators. Proceedings of Surveillance 6, Compiègne, paper $5.12 \mathrm{p}$.

[11] V.H. Vu, M. Thomas, F. Lafleur, May 2011, Operational Modal Monitoring of High Power Hydro-electrical Generators, Proceedings of IOMAC, Istanbul, paper 410 $8 \mathrm{p}$

[12] V.H. Vu, M. Thomas, A.A. Lakis, L. Marcouiller, ShortTime Autoregressive (STAR) Modeling for Operational Modal Analysis of Non-stationary Vibration. Chapter 3 on book: Vibration and structural acoustic analysis, Springer, 2011, pp. 59-77

[13] Vu V.H., M. Thomas, A.A. Lakis and L. Marcouiller, 2010, On line Time-Modal monitoring of non stationary systems, Revue Internationale sur l'Ingénierie des Risques Industriels (JI-IRI), 3, pp. 45-65

[14] V.H. Vu, M. Thomas, 28 Août 2013, Uncertainties on Modal Parameters by Operational Modal Analysis, Congrès Français de la Mécanique, Bordeaux, 6 p 